\title{
Gerenciamento da Infraestrutura de Comunicação baseado na Distribuição Gaussiana do Tempo de Contato entre Veículos e a Infraestrutura
}

\author{
Lucas D. Silva ${ }^{1}$, Fernanda S. H. Sumika ${ }^{3}$, Daniel L. Guidoni ${ }^{3}$, João F. M. Sarubbi ${ }^{4}$, \\ Cristiano G. Pitangui ${ }^{2}$, Cristiano M. Silva ${ }^{1,2}$ \\ ${ }^{1}$ Universidade Federal de Ouro Preto (DECOM/UFOP) - MG - Brasil \\ ${ }^{2}$ Universidade Federal de São João del-Rei (DTECH/UFSJ) - MG - Brasil \\ ${ }^{3}$ Universidade Federal de São João del Rei (DCOMP/UFSJ) - MG - Brasil \\ ${ }^{4}$ Centro Federal de Educação Tecnológica (DECOM/CEFET) - MG - Brasil \\ lucas.dinizaluno.ufop.edu.br, joaoddecom. cefetmg.br \\ \{fsumika, guidoni, pitangui.cristiano, cristiano\}@ufsj.edu.br
}

\begin{abstract}
Seeking novel solutions for urban mobility seems paramount for 21 st century society. This work discusses the performance of vehicular networks relying on the Delta Network. Delta characterizes the connectivity of the vehicular network by the percentage of travel time in which the vehicles are connected to the infrastructure. This work presents a novel deployment strategy based on the Gaussian distribution of the percentage of the trip duration in which vehicles are covered by the infrastructure. Roadside units are distributed throughout the urban area through a heuristic based on weights assigned according to the trips that are covered by the new roadside unit. The most important gain of this approach is that the resulting coverage pattern favors most of the fleet experiencing similar connectivity, while vehicles with very low or very high connectivity become the exception. Results show that the strategy promotes the distribution of contact time according to the Gaussian distribution.
\end{abstract}

Resumo. Buscar novas soluções para a mobilidade urbana torna-se primordial para a sociedade do século XXI. Este trabalho discute o desempenho de redes veiculares através da Rede Delta. Delta caracteriza a conectividade da rede veicular pela porcentagem de tempo de viagem em que os veículos estão conectados à infraestrutura. Este trabalho apresenta uma estratégia de deposição para redes veiculares baseada na distribuição gaussiana do percentual da viagem em que veículos estão cobertos pela infraestrutura. Unidades de comunicação são distribuídas pela área urbana através de uma heurística baseada em pesos atribuídos de acordo com as viagens que são cobertas pela nova unidade. Como principal benefício dessa abordagem, o padrão de cobertura resultante favorece que a maior parte da frota experimente conectividade semelhante, enquanto que veículos com baixíssima ou altíssima conectividade se tornam exceção. Os resultados indicam que a estratégia promove a distribuição de tempos percentuais de contato de veículos com a infraestrutura em acordo com a distribuição gaussiana. 


\section{Introdução}

Nas últimas décadas, o crescimento da população urbana tem sido notável. Atualmente, $50 \%$ da população vive em áreas urbanas, e previsões indicam que até 2050 esse número chegará a 70\% [Prospects, 2007]. Hoje, 64\% de todas as viagens feitas estão dentro de ambientes urbanos e a quantidade total de quilômetros urbanos percorrida deverá triplicar até 2050 [Van Audenhove et al., 2014]. Assim, buscar novas soluções para a mobilidade urbana torna-se primordial para a sociedade do século XXI. Veículos autônomos [Lozano-Perez, 2012] e compartilhamento de veículos [Katzev, 2003] tendem a assumir cada vez mais os deslocamentos diários nos centros urbanos. Gradualmente, carros tendem a assumir o papel de serviços contratados, ao invés de bens de consumo, moldando uma nova indústria automobilística, influenciando a forma como são projetados, produzidos e comercializados.

A tecnologia de comunicação embarcada em veículos assumirá, cada vez mais, caráter essencial. Sistemas de navegação e automação do veículo podem se integrar à via para aumento da eficiência energética, promovendo a regulação do tráfego, mitigação de colisões e sincronização de viagens, fazendo com que a frota em operação seja minimizada em relação à demanda por deslocamentos. A integração digital dos veículos à infraestrutura viária e às demais entidades do sistema de trânsito numa rede única de comunicação permitirá, em fato, que a própria infraestrutura se reorganize. Vias inteligentes podem alterar sua velocidade permitida, barrar a permissão para estacionar, mudar a programação semafórica [Silva et al., 2015a] e, até mesmo, alterar seu sentido de tráfego, implementando corredores dinâmicos de breve duração para escoar rapidamente excedentes de tráfego que poderiam acarretar congestionamentos.

Funcionalidades com esse nível de criticidade dependem do projeto e disponibilização de redes de comunicação específicas e geridas por autoridades governamentais, com acesso controlado e altos níveis de confiabilidade e segurança. Diante disso, este trabalho aprofunda nosso estudo sobre estratégias para o projeto de redes veiculares infraestruturadas apresentando uma heurística baseada em pontos (scores) para definir os locais onde as unidades de comunicação (antenas) veicular devem ser implantadas numa cidade, de forma a se garantir metas pré-definidas de desempenho da rede. O desempenho é mensurado a partir da Rede Delta [Silva and Meira Jr., 2015], que caracteriza a conectividade da rede veicular pela porcentagem de tempo de viagem em que os veículos estão conectados à infraestrutura depositada ao longo da malha viária. A heurística considera uma estratégia baseada na distribuição gaussiana dos tempos de contatos de veículos com a infraestrutura.

Como principal benefício dessa abordagem, o padrão de cobertura resultante favorece que a maior parte da frota experimente conectividade semelhante, enquanto que veículos com baixíssima ou altíssima conectividade se tornam exceção. Os experimentos consideram o rastro de mobilidade realístico da cidade de Colônia [Uppoor et al., 2014]. Os resultados demonstram que a heurística atinge seu objetivo, promovendo a distribuição de tempos percentuais de contato de veículos com a infraestrutura em acordo com a distribuição gaussiana. A estratégia é comparada com a aplicação da mesma heurística usando uma distribuição de probabilidades uniforme, bem como a deposição da infraestrutura nos locais de maior tráfego.

Este trabalho encontra-se organizado da seguinte forma: A seção 2 apresenta os 
trabalhos relacionados. A seção 3 revisa a Rede Delta. A seção 4 apresenta a estratégia gaussiana. A seção 5 apresenta as estratégias usadas como baseline. A seção 6 apresenta os experimentos realizados. A seção 7 conclui o trabalho.

\section{Trabalhos Relacionados}

A literatura apresenta várias estratégias para o planejamento da infraestrutura de comunicação para redes veiculares. Uma descrição detalhada desses esforços é apresentada por Silva et al. [2017]. A probabilidade de contato é considerada nos seguintes trabalhos. Zheng et al. [2010] apresentam a avaliação da estratégia de implantação considerando a oportunidade de contato. A métrica mensura a fração da distância (ou tempo) que o veículo está em contato com a infraestrutura, enquanto que Lee and Kim [2010] propõem uma heurística gulosa para alocar a infraestrutura com o objetivo de melhorar a conectividade entre veículos, ao mesmo tempo em que reduzem suas desconexões, e cada interseção é considerada como uma potencial posição para uma unidade de comunicação. Pereira et al. [2018] apresentam uma modelagem multi-objetivo do planejamento de infraestrutura para a distribuição de streaming em redes veiculares.

Xie et al. [2013] abordam o problema de alocação de unidades de comunicação assumindo conhecimento prévio da trajetória de cada veículo. Baseando-se em dados históricos, os autores propõem um modelo probabilístico para inferir as localizações das unidades de comunicação. Já, Chi et al. [2013] consideram uma estratégia que distribui as unidades de comunicação tão equilibradamente quanto possível. No entanto, os autores comparam seus resultados com uma estratégia de alocação aleatória, que incorre em um desempenho muito baixo. De forma complementar, Liu et al. [2013] propõem uma estratégia de alocação de unidades de comunicação para a transferência de arquivos em redes veiculares modelando o encontro entre veículos e unidades de comunicação como uma cadeia da Markov homogênea de tempo contínuo.

Trullols et al. [2010] e Cataldi and Harri [2011] propõem a modelagem de alocação de unidades de comunicação como um problema de máxima cobertura, assumindo-se a premissa de conhecimento prévio das trajetórias dos veículos. Essa premissa é relaxada nos trabalhos [Silva et al., 2014, 2015b, 2016], que propõem modelos de complexidades crescentes usando apenas a taxa de migração de veículos entre regiões. Estudos analíticos também são encontrados na literatura. Bazzi et al. [2011] abordam os sistemas celulares como a solução mais viável a curto prazo para coletar mensagens informativas de veículos para um centro de controle remoto. O trabalho propõe um modelo matemático para avaliar o impacto do serviço imaginado na capacidade e cobertura de sistemas celulares em cenários simplificados. Os resultados mostram que a aquisição de pacotes pequenos e freqüentes de veículos é afetada por interferências mais do que outros serviços, como o serviço de voz.

Finalmente, o trabalho apresentado por Zheng et al. [2009] serve como inspiração para a Rede Delta [Silva and Meira Jr., 2015] revisada na seção 3. Os autores propõem a Cobertura Alpha para fornecer garantias no intervalo de interconexão medido em termos de distância percorrida. Uma rede veicular é considerada de cobertura $\alpha$ se um veículo percorrendo qualquer caminho de comprimento $\alpha$ passa, ao menos, por uma unidade de comunicação infraestruturada. Já a Rede Delta [Silva and Meira Jr., 2015] considera tempo de deslocamento, ao invés de distância percorrida. 
Diferentemente das abordagens anteriores, a estratégia aqui proposta considera a implantação de unidades de comunicação (antenas) de forma a realizar uma distribuição gaussiana dos tempos em que veículos permanecem conectados à infraestrutura.

\section{Avaliando a Conectividade Veicular Através da Métrica Rede Delta}

A Rede Delta Silva and Meira Jr. [2015] avalia o tempo de conexão dos veículos em relação à duração viagem, caracterizando uma dimensão do desempenho da rede veicular. Sua representação é dada por $\Delta_{\rho_{2}}^{\rho_{1}}$, onde o parâmetro $\rho_{1}$ representa a porcentagem de tempo de viagem conectado, enquanto que $\rho_{2}$ indica porcentagem de veículos que experimentam a conectividade indicada por $\rho_{1}$. Sendo assim, uma rede veicular torna-se $\Delta_{\rho_{2}}^{\rho_{1}}$ quando $\rho_{2}$ por cento dos veículos experimentam conexão com unidades de comunicação (antenas) durante $\rho_{1}$ por cento da viagem.

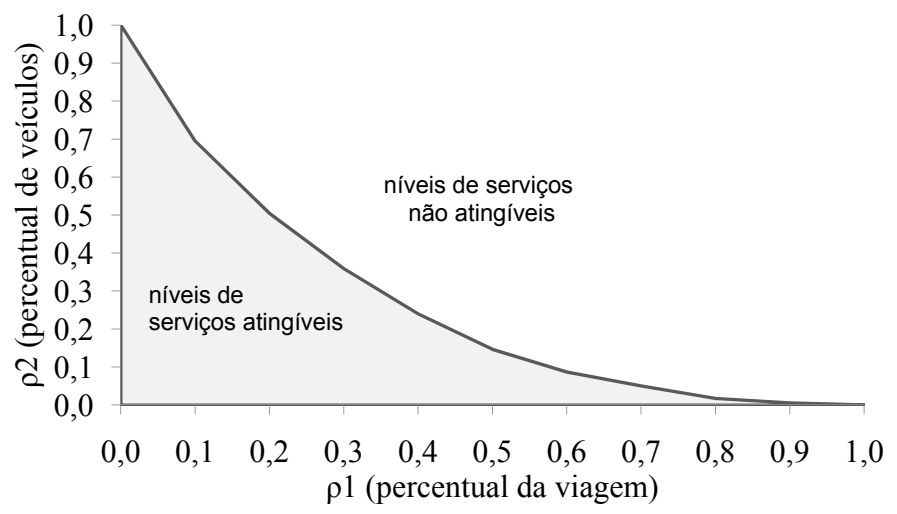

Figura 1. Figura apresenta a Rede Delta que representa a relação entre $\rho_{1}$ (percentual de duração da viagem sob cobertura) versus $\rho_{2}$ (percentual de veículos que recebem tal cobertura). Fonte: [Silva and Meira Jr., 2015].

A figura 1 exemplifica a métrica Delta. O eixo $x$ representa $\rho_{1}$ (porcentagem da viagem conectado), enquanto que o eixo $y$ indica $\rho_{2}$ (porcentagem de veículos conectados). Assim, Delta indica a relação entre $\rho_{1}$ e $\rho_{2}$ para toda a frota que se desloca numa dada região. A área abaixo da curva representa os níveis de conectividade atingíveis pela rede. Fora da curva, os níveis de conectividade não podem ser alcançados. Como a Rede Delta descreve uma relação de conectividade, ela pode ser utilizada para o planejamento e expansão de redes veiculares de forma a se atingir níveis pré-definidos de conectividade, uma aplicação fundamental para se garantir que a rede suporte as aplicações para as quais foi projetada.

\section{Estratégia Gaussiana: Promovendo uma Distribuição Natural do Percentual de Tempo Viajado em que Veículos estão Cobertos}

Estratégias de solução locais para a Rede Delta já foram apresentadas nos trabalhos [Sarubbi and Silva, 2016; Silva and Meira Jr, 2015]. No entanto, essas soluções focam na descoberta dos locais que precisam receber cobertura de forma a se obter a configuração de unidades de comunicação (antenas) para uma única tupla $\left\{\rho_{1}, \rho_{2}\right\}$, fazendo com que os demais pontos da curva Delta surjam como consequência da descoberta desse ponto inicial. No entanto, encontrar soluções para um único par $\left\{\rho_{1}\right.$, $\left.\rho_{2}\right\}$ pode não garantir boa eficiência para outras combinações de $\left\{\rho_{1}, \rho_{2}\right\}$. 


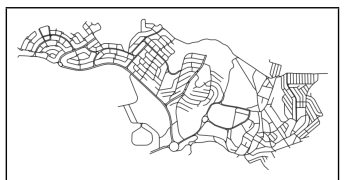

(a) Malha Viária.

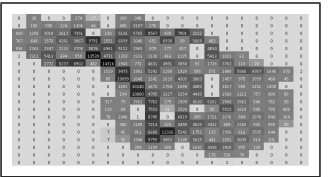

(b) Grade $20 \times 20$.

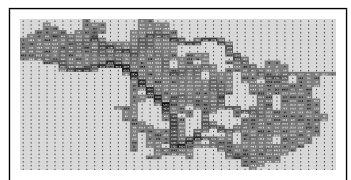

(c) Grade $40 \times 40$.

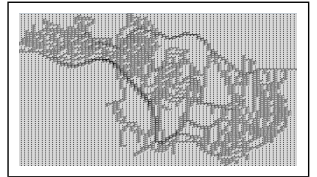

(d) Grade $80 \times 80$.

Figura 2. Particionamento da malha viária em células urbanas.

Assim, essa investigação é expandida para se obter todos os pontos da curva. Para tanto, considera-se uma estratégia baseada em pontuações (scores) atribuídas para áreas da malha viária. Em fato, malhas viárias podem assumir topologias arbitrárias que resultam da forma de ocupação, relevo, dentre outros aspectos. Esse trabalho propõe uma estrutura baseada em grade ( grid), conforme mostrado na figura 2, onde a cidade é dividida em células adjacentes de mesmo tamanho. Este tamanho pode variar de acordo com a precisão e cobertura esperada. A escolha das células que receberá cobertura se dá a partir da heurística geral abaixo apresentada em [Silva et al., 2018], que considera soluções de uma perspectiva global para toda a curva Delta.

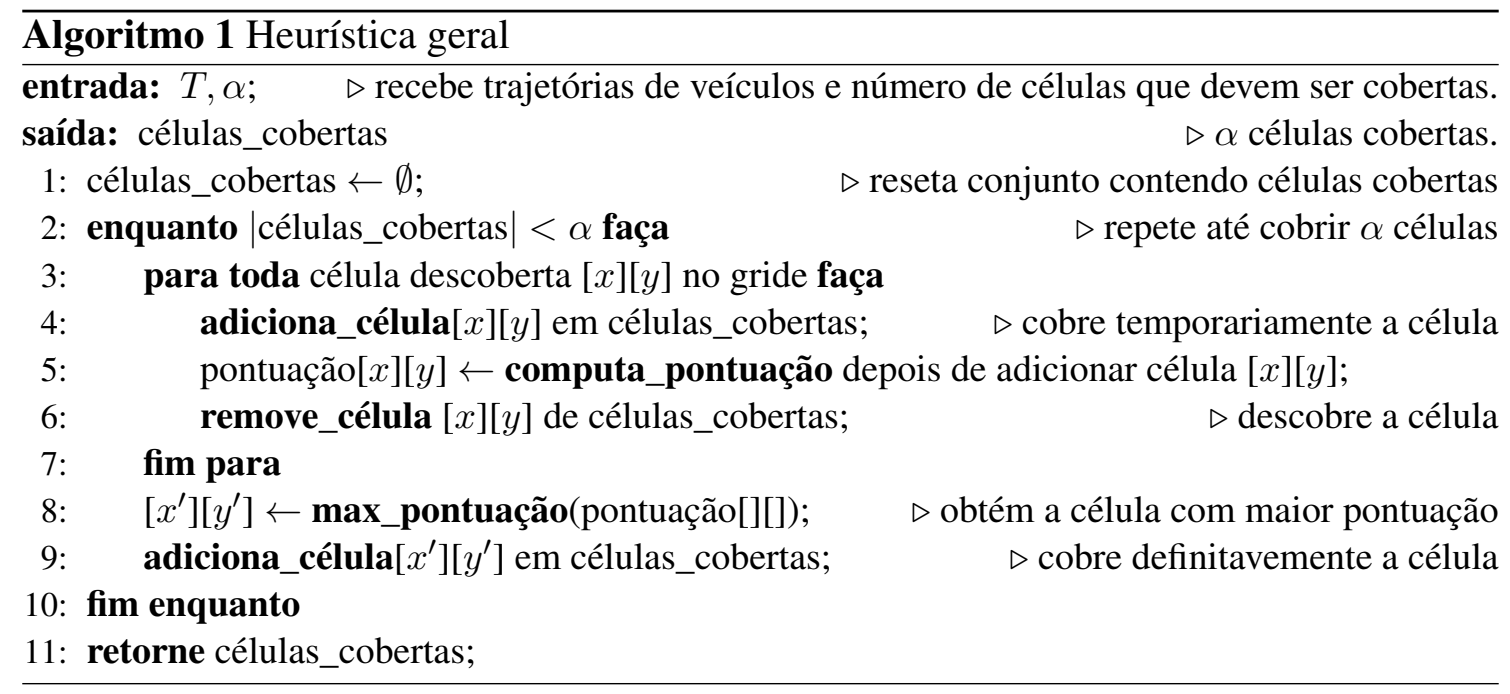

O algoritmo 1 apresenta a heurística gulosa geral que recebe como entrada (i) o rastro de veículos $(T)$ e (ii) o número de células disponíveis a serem cobertas. O algoritmo inicia com um conjunto vazio contendo as células a serem cobertas (linha 1). Como nenhuma célula é coberta inicialmente, a heurística começa cobrindo temporariamente a célula $[0,0]$ e avalia o desempenho da rede em termos de $\left\{\rho_{1}, \rho_{2}\right\}$. Em seguida, ela remove a cobertura de $[0,0]$ e move para $[0,1]$ e recalcula o desempenho da rede. Esse procedimento segue até avaliar todos os locais do gride (linhas 3-7). Em seguida, seleciona a célula que apresenta a pontuação mais alta. A célula que apresenta a pontuação mais alta é selecionada permanentemente para receber cobertura (linhas 8-9). Então, a heurística faz um laço até selecionar $\alpha$ células cobertas.

O cálculo de pontuação (linha 5) merece atenção especial, pois permite personalizar a forma de seleção das células, determinando o padrão de cobertura obtido. Tem-se a oportunidade de impor propriedades específicas na rede veicular, como selecionar o tipo de viagem que pretendemos priorizar e como a conectividade é 
distribuída entre veículos. O estudo aqui apresentado considera pesos atribuídos com base numa distribuição normal padrão sobreposta ao eixo $x$ da figura 1, isto é, $\rho_{1}$ que representa o percentual de tempo viajado. A distribuição normal padrão é centrada no ponto $\rho_{1}=0.5$, atribuindo-se maior pontuação para células que colaboram para que veículos estejam cobertos durante $50 \%$ do tempo viajado. Assim, esse trabalho avalia uma nova estratégia para a alocação de pesos baseada na distribuição padrão normal. A personalização da cobertura se dá através da valoração diferenciada de cada região do eixo $x$ do gráfico da Rede Delta. Dito de outra forma, personaliza-se o perfil de cobertura que a rede deve ofertar.

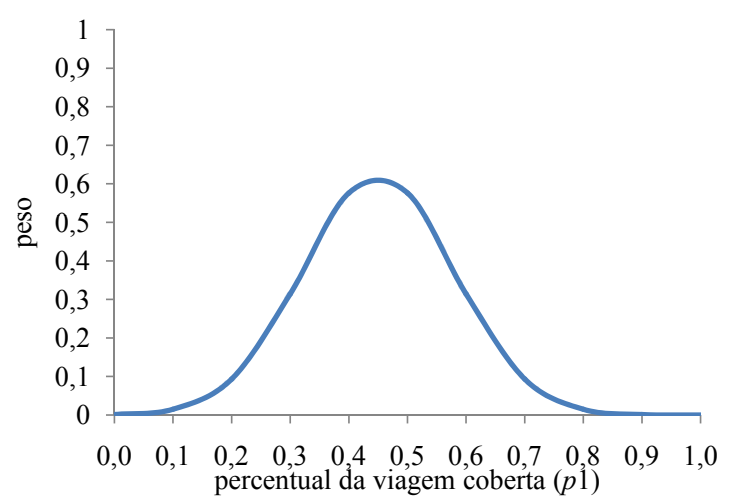

(a) Estratégia Gaussiana (proposta)

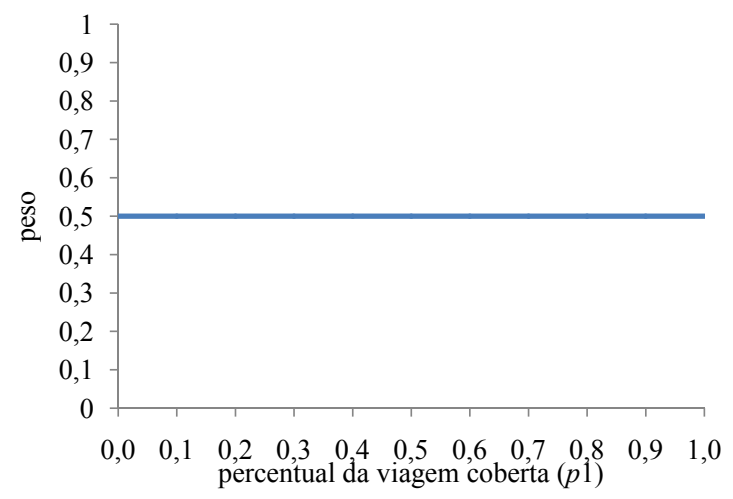

(b) Estratégia Balanceada (baseline)

Figura 3. Distribuição de pesos para implantação de unidades de comunicação, de acordo com o percentual da viagem coberto. A figura (a) apresenta a estratégia proposta, onde a pontuação máxima é atribuída para células que contribuem para viagens cobertas durante $50 \%$ do percurso, tipo de cobertura que será priorizada pela heurística. A figura (b) apresenta a distribuição de pesos considerada como baseline, onde todas as viagens apresentam igual prioridade.

A figura 3(a) apresenta a distribuição de pesos considerada. O eixo $x$ indica a duração da viagem. O eixo y indica o peso considerado. Essa curva é uma distribuição normal padrão com média deslocada para o ponto $x=0.5$. Já a figura 3(b) apresenta uma distribuição de pesos uniformemente distribuída ao longo do eixo $x$. Essa é a distribuição de pesos adotada pela estratégia balanceada, usada como baseline. A distribuição uniforme atribui pesos idênticos para todas as viagens, não havendo priorização. Como pode ser percebido pela figura 3(a), a estratégia gaussiana atribui pesos maiores para valores localizados nas vizinhanças de $\rho_{1}=0.5$. Lembre-se que $\rho_{1}$ indica "duração da viagem sob cobertura". Assim, a atribuição de pesos gaussiana acaba por incentivar a cobertura de áreas que resultem em mais viagens atingindo cobertura durante $50 \%$ do tempo viajado do veículo.

\section{Baseline}

São consideradas duas estratégias para se avaliar o desempenho da heurística proposta. A estratégia intuitiva cobre as células que possuem maior tráfego de veículos até que se atinja os níveis desejados de desempenho da rede. Já, a estratégia balanceada considera a mesma heurística geral apresentada no algoritmo 1 conjugada com a distribuição uniforme de pesos apresentada na figura 3(b), onde todos os tipos de viagem recebem a 
mesma priorização. A estratégia balanceada é descrita em detalhes em [Silva et al., 2018], sendo a maximização da área sob a curva Delta sua característica mais interessante.

\subsection{Estratégia balanceada: distribuição uniforme de pesos, independentemente do percentual de duração da viagem sob cobertura: uma alternativa para maximizar a área sob a curva Delta}

Suponha apenas dois veículos viajando ao longo da malha viária dividida em 5 células. Suponha, também, que tais veículos permaneçam a mesma quantidade de tempo dentro de cada célula, e que existam antenas capazes de cobrir apenas duas células $(\alpha=2)$. $\mathrm{O}$ veículo $v_{1}$ cruza as células $\{1,2\}$, enquanto que o veículo $v_{2}$ cruza as células $\{1,3,4,5\}$.

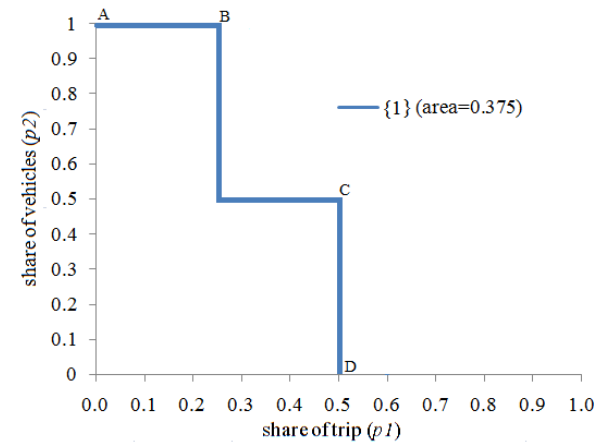

(a) 1a iteração da estratégia balanceada.

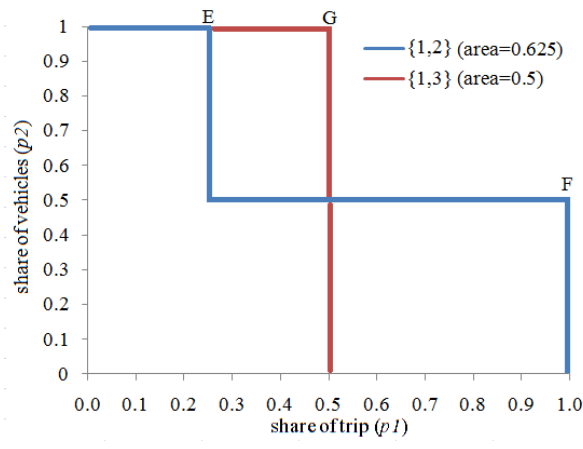

(b) 2a iteração da estratégia balanceada.

Figura 4. Exemplo didático sobre como a estratégia balanceada funciona. A figura (a) apresenta a Rede Delta depois de cobrir a célula 1. A figura (b) apresenta a Rede Delta considerando duas opções: a) cobrindo célula 2; b) cobrindo célula 3. Como cobrir a célula 2 leva a uma área maior sob a curva, a célula 2 é selecionada. Fonte: Silva et al. [2018].

$$
\begin{gathered}
v_{1}=\{1,2\} ; \\
v_{2}=\{1,3,4,5\} ;
\end{gathered}
$$

Como a célula 1 é cruzada por ambos os veículos, ela intuitivamente maximiza a área sob a curva Delta. Depois de cobrir a célula 1, têm-se o veículo $v_{1}$ coberto durante $50 \%$ da viagem e o veículo $v_{2}$ coberto durante $25 \%$ da viagem. A figura 4 (a) plota a Rede Delta para este cenário hipotético. O ponto $A$ é $\left(\rho_{1}=0,00, \rho_{2}=1,00\right)$, ou seja, $100 \%$ dos veículos são cobertos durante $0 \%$ da duração da viagem (veículos $v_{1}$ e $v_{2}$ ). $\mathrm{O}$ ponto $B$ é $\left(\rho_{1}=0,25, \rho_{2}=1,00\right)$, ou seja, $100 \%$ dos veículos são cobertos durante $25 \%$ da duração da viagem (veículos $v_{1}$ e $\left.v_{2}\right)$. O ponto $C$ é $\left(\rho_{1}=0.50, \rho_{2}=0.50\right)$, ou seja, $50 \%$ dos veículos são cobertos durante $50 \%$ da duração da viagem (veículo $v_{1}$ ). O ponto $D$ é $\left(\rho_{1}=0,50, \rho_{2}=0,00\right)$, ou seja, nenhum veículo é coberto por mais de $50 \%$ da duração da viagem.

\subsection{Estratégia Intuitiva}

Já a estratégia intuitiva cobrir locais seguindo a ordem de popularidade. A célula mais popular é coberta primeiro. Recebe como entrada o volume de tráfego por célula $(V)$, e o número de células a serem selecionadas $(\alpha)$, conforme apresentado no algoritmo 2. 


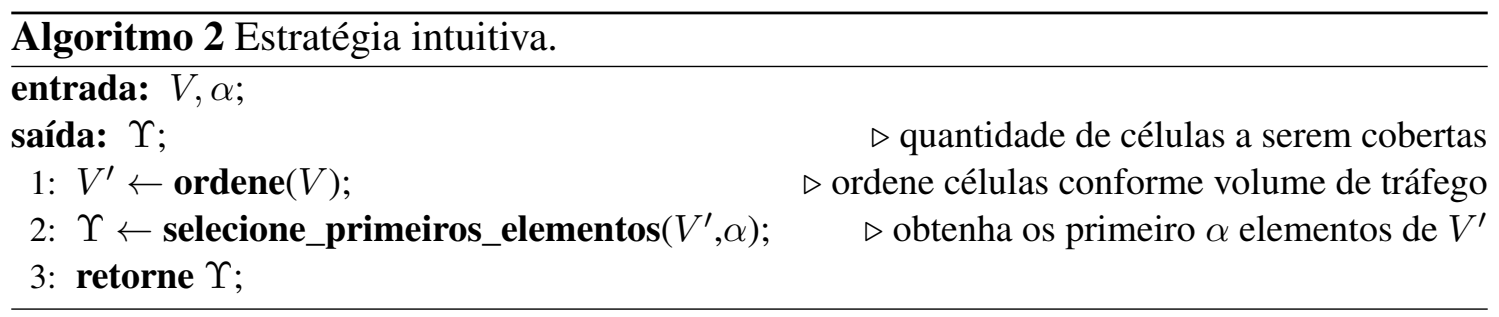

\section{Avaliação Experimental}

Os experimentos são conduzidos usando-se o rastro de mobilidade veicular da cidade de Colônia, Alemanha [Uppoor et al., 2014] ${ }^{1}$ composto por 10 mil segundos de tráfego e mais de 75 mil veículos. A malha viária foi particionada num gride de $100 \times 100$ células urbanas, e avalia-se o desempenho da rede considerando a cobertura de $[5 \%, 10 \%, 15 \%$ e $20 \%$ ] da malha viária que possui tráfego.

\subsection{Avaliando a QoS provida pela rede}

Nesse estudo, busca-se avaliar a QoS provida pela rede a partir da deposição de unidades de comunicação proposta, comparada às duas estratégias de baseline. Cada estratégia deposita unidades de comunicação de forma cobrir $5 \%, 10 \%$ ou $15 \%$ da área urbana. A figura 5(a) plota a QoS apurada em termos da Rede Delta (percentual da viagem em que veículo viaja com cobertura (eixo $x$ ) versus quantidade de veículos (eixo $y$ ). A curva azul apresenta os resultados da estratégia gaussiana (proposta). Nota-se que essa estratégia oferece uma quantidade similar de veículos experimentando cobertura quando comparada à estratégia intuitiva (curva vermelha) para $\rho_{1}<0,2$ (eixo $x$ da figura 5(a)).

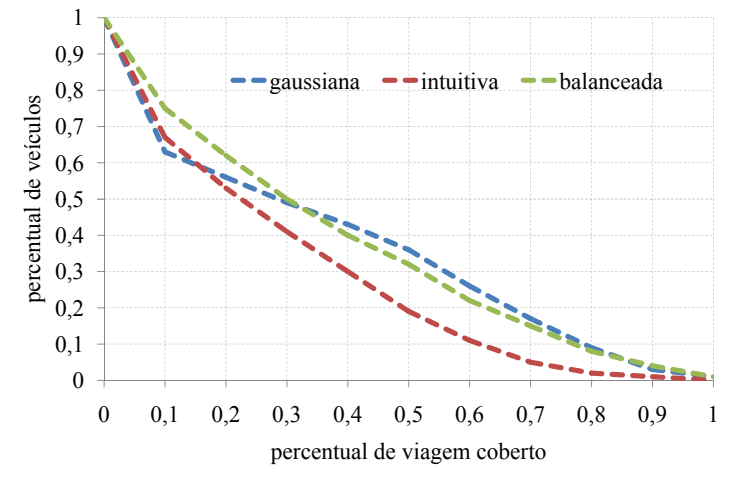

(a) cobertura de $5 \%$ da malha viária.

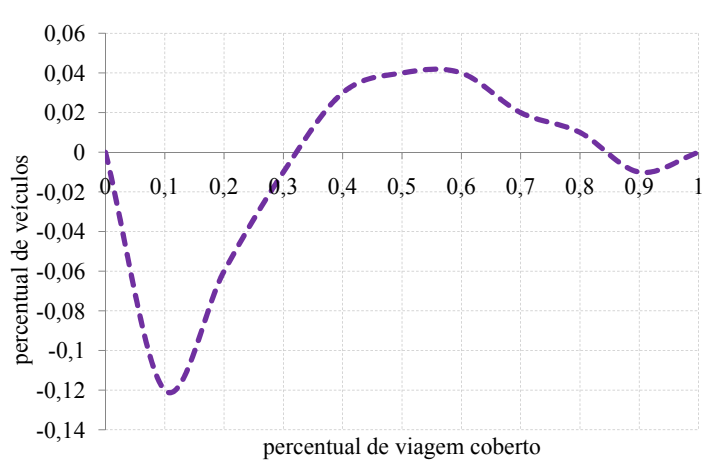

(b) diferença entre gaussiana e balanceada (5\%).

Figura 5. Cenário de cobertura de $5 \%$ da malha viária. A figura (a) apresenta a Rede Delta para cada uma das três estratégias: a) gaussiana (cor azul), b) intuitiva (cor vermelha), a) balanceada (cor verde). 0 eixo $x$ indica o percentual da viagem coberto $\left(\rho_{1}\right)$, enquanto que o eixo $y$ indica o percentual de veículos $\left(\rho_{2}\right)$. A figura (b) plota a diferença gaussiana menos balanceada.

Nesse intervalo, a estratégia balanceada (cor verde) vence as demais. No entanto, quanto mais próximo da metade do eixo $x$, melhor é o resultado apresentado pela estratégia gaussiana. Isso deve-se ao esquema de pontuação gaussiano proposto que valoriza a cobertura de células que resultem em viagens com cobertura de $50 \%$ do tempo

\footnotetext{
${ }^{1}$ Disponível em: http://kolntrace.project.citi-lab.fr/.
} 


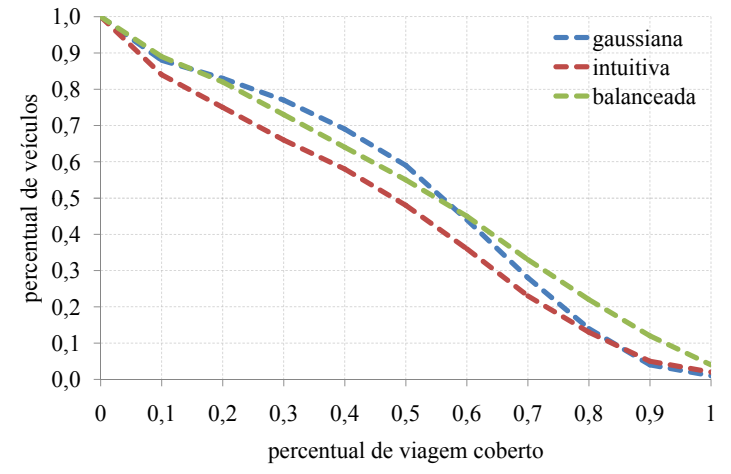

(a) cobertura de $10 \%$ da malha viária.

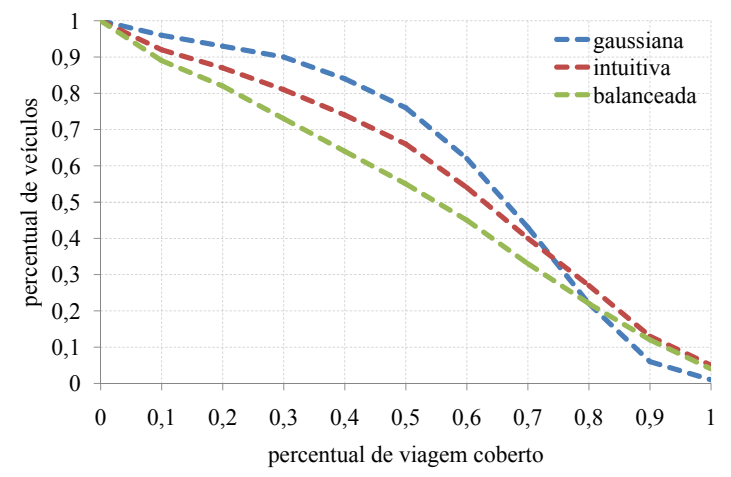

(c) cobertura de $15 \%$ da malha viária.

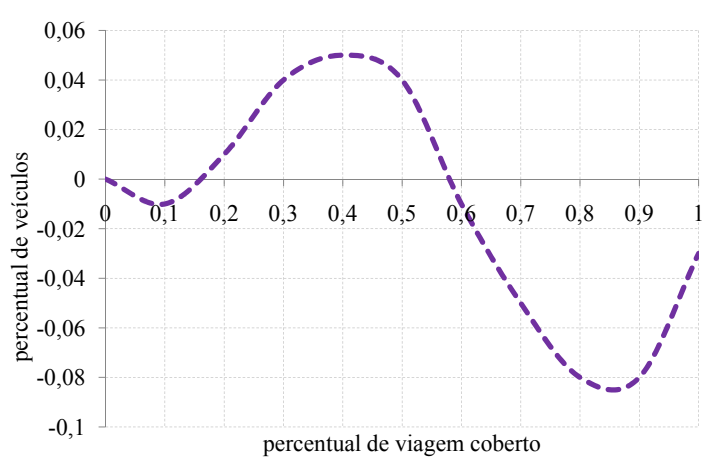

(b) diferença entre gaussiana e balanceada (10\%).

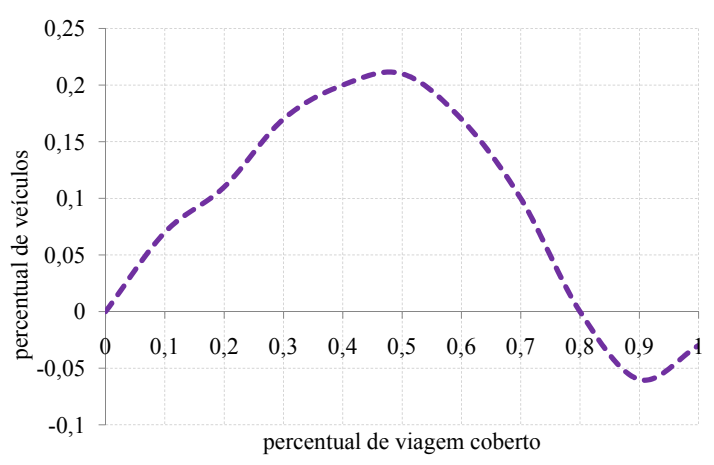

(d) diferença entre gaussiana e balanceada (15\%).

Figura 6. As figuras (a) e (b) apresentam o cenário de cobertura de $10 \%$ da malha viária, enquanto que as figuras (c) e (d) apresentam a cobertura de $15 \%$ da malha. A estratégia gaussiana é representada na cor azul, intuitiva na cor vermelha e balanceada na cor verde. 0 eixo $x$ indica o percentual da viagem coberto $\left(\rho_{1}\right)$, enquanto que o eixo $y$ indica o percentual de veículos $\left(\rho_{2}\right)$.

de deslocamento. Por outro lado, a estratégia balanceada maximiza a área abaixo da curva Delta [Silva et al., 2018]. Já a figura 5(b) plota apenas a diferença de cada ponto da estratégia gaussiana subtraído do mesmo ponto na estratégia balanceada. Curva acima do eixo $x$ indica que a estratégia gaussiana apresentou mais veículos atendidos. Ao analisar o gráfico, percebe-se que a estratégia gaussiana apresenta mais veículos experimentando cobertura de $30 \%$ até $85 \%$ da viagem (veja o eixo $x$ da figura 5(b) entre os valores 0.3 e 0.8 ). Note que o pico da diferença é atingido exatamente em $x=0.5$.

As figuras 6(a) e 6(b) apresentam o cenário onde $10 \%$ da malha viária é coberto. Nota-se que a estratégia intuitiva apresenta menor quantidade de veículos para, praticamente, toda a curva. A estratégia gaussiana se destaca no intervalo $0.2 \leq$ $x \leq 0.6$. Para $x>0.6$, a estratégia balanceada apresenta melhor desempenho. A visão comparativa (gaussiana menos balanceada) é apresentada na figura 6(b). O fato de não se notar um pico no ponto $x=0.5$ na figura $6(\mathrm{~b})$ deve-se ao fato da estratégia balanceada ter obtido um bom volume de veículos cobertos nessa faixa em decorrência da maximização da área sob a curva Delta, obra do acaso, visto que a estratégia não possui como objetivo tal comportamento. Finalmente, as figuras 6(c) e 6(d) apresentam o cenário de cobertura de $15 \%$ da malha viária, onde nota-se, novamente maior volume de veículos cobertos pela estratégia gaussiana quando $x=0.5$ na figura $6(\mathrm{~d})$. 


\subsection{Layout de Cobertura}

Essa seção apresenta o layout de disposição das células cobertas. A figura 7(a) mostra o tráfego na cidade de Colônia, Alemanha. A intensidade da cor é diretamente proporcional ao tráfego. O layout decorrente da estratégia intuitiva é apresentados nas figuras 7(b)-7(d). De forma similar, o layout decorrente da estratégia balanceada é apresentado nas figuras $7(\mathrm{e})-7(\mathrm{~g})$. Finalmente, a estratégia gaussiana é indicada nas figuras 7(h)-7(j).

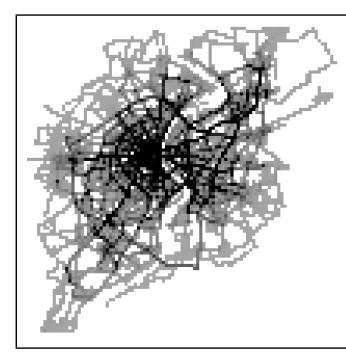

(a) Tráfego

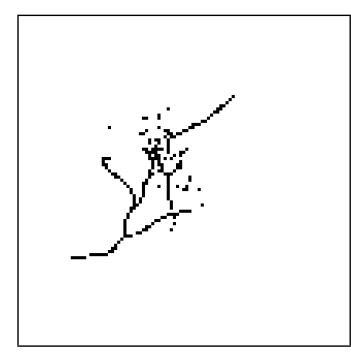

(b) intuitiva (5\%)

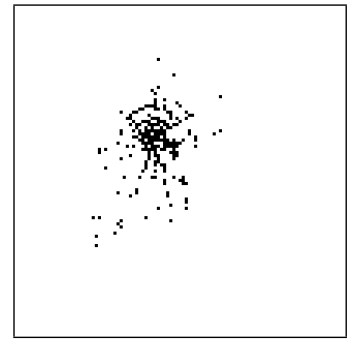

(e) balanceada (5\%)

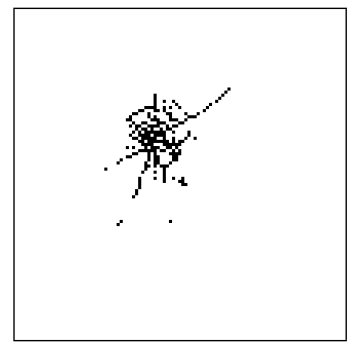

(h) gaussiana (5\%)

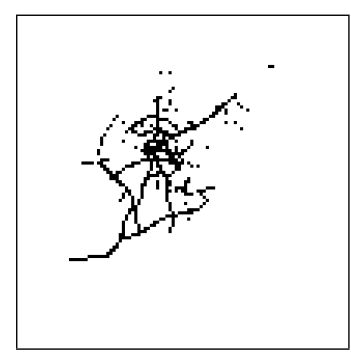

(c) intuitiva (10\%)

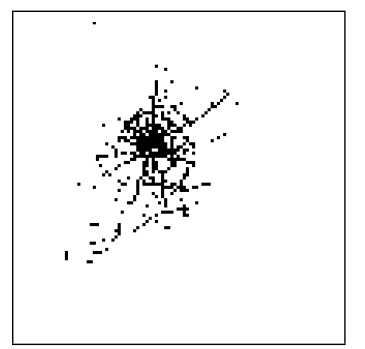

(f) balanceada (10\%)

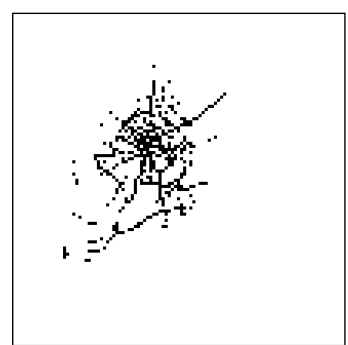

(i) gaussiana (10\%)

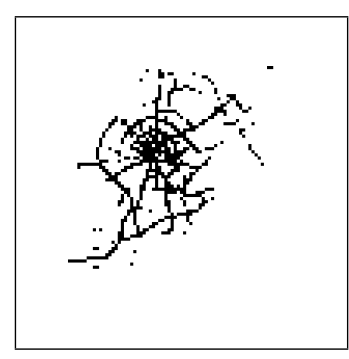

(d) intuitiva (15\%)

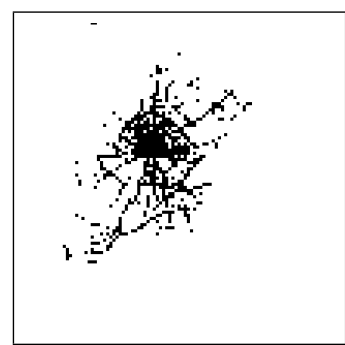

(g) balanceada (15\%)

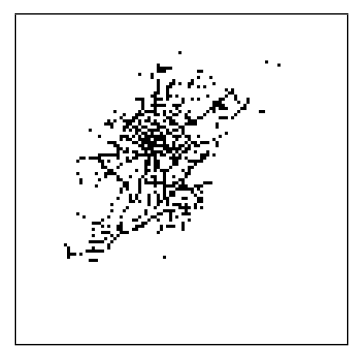

(j) gaussiana (15\%)

Figura 7. A figura 7(a) mostra o tráfego na cidade de Colônia, Alemanha. 0 layout decorrente da disposição de células cobertas pela estratégia intuitiva é apresentados nas figuras $7(b)-7(d)$. A estratégia balanceada está nas figuras $7(e)-7(g)$. A estratégia gaussiana é indicada nas figuras $7(\mathrm{~h})-7(\mathrm{j})$.Note que cada coluna agrupa o mesmo cenário, facilitando a comparação entre as estratégias.

Nota-se que a estratégia intuitiva segue os principais corredores de tráfego da malha viária. Já a estratégia balanceada realiza a cobertura em círculos concêntricos a partir do epicentro de tráfego, afinal seu objetivo é maximizar a área sob a curva Delta. Já a estratégia gaussiana mescla características de ambas. Veja que o corredor de tráfego posterior existente na figura 7(b) não existe na figura 7(e), mas aparece na figura 7(h). Por outro lado, o layout geral da estratégia gaussiana herda a característica de espalhamento e cobertura concêntrica da estratégia balanceada. 


\section{Conclusão}

Este trabalho discute o desempenho de redes veiculares em termos da métrica Rede Delta. A Rede Delta caracteriza a conectividade da rede veicular pela porcentagem de tempo de viagem em que os veículos estão conectados às unidades de beira de estrada. Em resumo, este artigo revisa o conceito da Rede Delta e estende seu estudo através da apresentação de uma estratégia baseada na distribuição gaussiana do tempo de contato entre veículos e a infraestrutura. Considera-se o rastro realístico de mobilidade veicular da cidade de Colônia, Alemanha, com a malha viária particionada em uma estrutura semelhante a uma grade de dimensões $100 \times 100$, resultando em células urbanas de dimensão $270 \mathrm{~m} \times 260 \mathrm{~m}$ por onde trafegam mais de 75 mil veículos.

A definição da pontuação de cada célula baseia-se no número de veículos cobertos em relação à distância percorrida. Como principal benefício dessa abordagem, o padrão de cobertura resultante favorece que a maior parte da frota experimente conectividade semelhante, enquanto que veículos com baixíssima ou altíssima conectividade se tornam exceção. Os resultados demonstram que a heurística atinge seu objetivo, promovendo a distribuição de tempos percentuais de contato de veículos com a infraestrutura em acordo com a distribuição gaussiana. Como baseline, a estratégia é avaliada com a aplicação da mesma heurística usando uma distribuição de probabilidades uniforme, bem como a intuitiva deposição da infraestrutura nos locais de maior tráfego.

Como trabalho futuro, pretendemos desenvolver modelos ótimos para a estratégia aqui proposta, bem como buscar novas estratégias para atribuir pontuações para células, gerando novas estratégias de implantação.

\section{Agradecimentos}

Esse trabalho foi parcialmente financiado por CNPq, CAPES, FAPEMIG, Departamento de Tecnologia da Universidade Federal de São João del-Rei (DTECH/UFSJ) e o Departamento de Computação da Universidade Federal de Ouro Preto (DECOM/UFOP).

\section{Referências}

Bazzi, A., Masini, B. M., and Andrisano, O. (2011). On the frequent acquisition of small data through rach in umts for its applications. IEEE Transactions on Vehicular Technology, 60(7):2914-2926.

Cataldi, P. and Harri, J. (2011). User/operator utility-based infrastructure deployment strategies for vehicular networks. In Vehicular Technology Conference (VTC Fall), 2011 IEEE, pages 1-5.

Chi, J., Jo, Y., Park, H., and Park, S. (2013). Intersection-priority based optimal rsu allocation for vanet. In Ubiquitous and Future Networks (ICUFN), 2013 Fifth International Conference on, pages 350-355.

Katzev, R. (2003). Car sharing: A new approach to urban transportation problems. Analyses of Social Issues and Public Policy, 3(1):65-86.

Lee, J. and Kim, C. (2010). A roadside unit placement scheme for vehicular telematics networks. In Kim, T.-h. and Adeli, H., editors, Advances in Computer Science and Information Technology, volume 6059 of Lecture Notes in Computer Science, pages 196-202. Springer Berlin Heidelberg. 
Liu, Y., Niu, J., Ma, J., and Wang, W. (2013). File downloading oriented roadside units deployment for vehicular networks. Journal of Systems Architecture, 59(10, Part B):938 - 946. Advanced Smart Vehicular Communication System and Applications.

Lozano-Perez, T. (2012). Autonomous robot vehicles. Springer Science \& Business Media.

Pereira, L. A. A., Luciano, M. C., Mariz, L. L., Ramos, B. V., Pitangui, C. G., and Silva, C. M. (2018). Um modelo multiobjetivo para alocação de unidades de comunicação para distribuição de conteúdos em redes veiculares. Workshop de Gerência e Operação de Redes e Serviços (WGRS $\left.S_{S} B R C\right), 23$.

Prospects, W. U. (2007). World urbanization prospects: The 2007 revision.

Sarubbi, J. F. M. and Silva, C. M. (2016). Delta-r: A novel and more economic strategy for allocating the roadside infrastructure in vehicular networks with guaranteed levels of performance. In NOMS 2016 - 2016 IEEE/IFIP Network Operations and Management Symposium, pages 665-671.

Silva, C. M., Aquino, A. L. L., and Meira Jr., W. (2014). Design of roadside infrastructure for information dissemination in vehicular networks. In Network Operations and Management Symposium (NOMS), 2014 IEEE, pages 1-8.

Silva, C. M., Aquino, A. L. L., and Meira Jr, W. (2015a). Smart Traffic Light for Low Traffic Conditions. Mobile Networks and Applications, pages 1-9.

Silva, C. M., Aquino, A. L. L. A., and Meira, Jr, W. (2015b). Deployment of roadside units based on partial mobility information. Computer Communications, 60(0):28 39.

Silva, C. M., Masini, B. M., Ferrari, G., and Thibault, I. (2017). A Survey on Infrastructure-Based Vehicular Networks. Mobile Information Systems, 2017, Article ID 6123868:28-56.

Silva, C. M. and Meira Jr, W. (2015). Design of roadside communication infrastructure with QoS guarantees. In 2015 IEEE Symposium on Computers and Communication (ISCC), pages 439-444.

Silva, C. M. and Meira Jr., W. (2015). Evaluating the Performance of Heterogeneous Vehicular Networks. In 2015 IEEE 82nd Vehicular Technology Conference (VTC2015-Fall), pages 1-5.

Silva, C. M., Meira Jr., W., and Sarubbi, J. F. M. (2016). Non-intrusive planning the roadside infrastructure for vehicular networks. IEEE Transactions on Intelligent Transportation Systems, 17(4):938-947.

Silva, C. M., Silva, L. D., Santos, L. A. L., Sarubbi, J. F. M., and Pitsillides, A. (2018). Broadening understanding on managing the communication infrastructure in vehicular networks: Customizing the coverage using the delta network. Future Internet, 11(1).

Trullols, O., Fiore, M., Casetti, C., Chiasserini, C., and Ordinas, J. B. (2010). Planning roadside infrastructure for information dissemination in intelligent transportation systems. Computer Communications, 33(4):432 - 442.

Uppoor, S., Trullols-Cruces, O., Fiore, M., and Barcelo-Ordinas, J. M. (2014). Generation and analysis of a large-scale urban vehicular mobility dataset. IEEE Transactions on Mobile Computing, 13(5):1061-1075.

Van Audenhove, F.-J., Korniichuk, O., Dauby, L., and Pourbaix, J. (2014). The Future of Urban Mobility 2.0: Imperatives to Shape Extended Mobility Ecosystems of Tomorrow. 
Xie, B., Xia, G., Chen, Y., and Xu, M. (2013). Roadside infrastructure placement for information dissemination in urban its based on a probabilistic model. In Network and Parallel Computing, volume 8147 of Lecture Notes in Computer Science, pages 322-331. Springer Berlin Heidelberg.

Zheng, Z., Lu, Z., Sinha, P., and Kumar, S. (2010). Maximizing the contact opportunity for vehicular internet access. In INFOCOM, 2010 Proceedings IEEE, pages 1-9.

Zheng, Z., Sinha, P., and Kumar, S. (2009). Alpha coverage: Bounding the interconnection gap for vehicular internet access. In INFOCOM 2009, IEEE, pages $2831-2835$. 